\title{
Balkanologie
}

Balkanologie Revue d'études pluridisciplinaires

Vol. XIII, $n^{\circ}$ 1-2 | 2011

Volume XIII Numéro 1-2

Lorain (Marie-Thérèse) et Lory (Bernard) éds, Guillaume Lejean. Voyages dans les Balkans (1857-1870)

Paris : Non-Lieu, 2011, 496 p. [coll. « Via Balkanica »]

\section{Nicolas Trifon}

\section{OpenEdition}

\section{Journals}

Édition électronique

URL : http://journals.openedition.org/balkanologie/2314

DOI : 10.4000/balkanologie.2314

ISSN : 1965-0582

\section{Éditeur}

Association française d'études sur les Balkans (Afebalk)

Référence électronique

Nicolas Trifon, «Lorain (Marie-Thérèse) et Lory (Bernard) éds, Guillaume Lejean. Voyages dans les Balkans (1857-1870) », Balkanologie [En ligne], Vol. XIII, n 1-2 | 2011, mis en ligne le 20 décembre 2011, consulté le 17 décembre 2020. URL : http://journals.openedition.org/balkanologie/2314 ; DOI : https:// doi.org/10.4000/balkanologie.2314

Ce document a été généré automatiquement le 17 décembre 2020.

(c) Tous droits réservés 


\title{
Lorain (Marie-Thérèse) et Lory (Bernard) éds, Guillaume Lejean. Voyages dans les Balkans (1857-1870)
}

Paris : Non-Lieu, 2011, 496 p. [coll. « Via Balkanica »]

\author{
Nicolas Trifon
}

\section{RÉFÉRENCE}

Lorain (Marie-Thérèse) et Lory (Bernard) éds, Guillaume Lejean. Voyages dans les Balkans (1857-1870), Paris : Non-Lieu, 2011, 496 p. [coll. « Via Balkanica »]

1 Parus dans des publications spécialisées - tel le Bulletin de la Société de géographie - ou destinées à un large public - la Revue des deux mondes, le Magasin pittoresque - les lettres, mémoires, rapports de mission issus des archives et articles de Guillaume Lejean (1824-1871) ont été réunis pour la première fois dans un épais volume qui vient de paraitre aux éditions Non Lieu. L'éditeur rend enfin disponibles les travaux d'un auteur qui a connu son heure de gloire du temps de Napoléon III et qui demeure une référence pour les géographes et les historiens ${ }^{1}$. L'introduction est signée par la biographe du voyageur-géographe; le choix des matériaux, leur présentation, la postface et l'appareil de notes, très fourni, a été rédigé par l'historien Bernard Lory.

Il s'agit d'un travail d'édition remarquable à tout point de vue. Pour rendre accessible une œuvre aussi disparate, rédigée dans une langue dont certaines tournures peuvent surprendre de nos jours, et surtout pour identifier les faits, rectifier les erreurs, rendre intelligibles les ethnonymes, toponymes et autres anthroponymes orthographiés à l'ancienne ou sortis d'usage depuis longtemps, pour préciser certaines références, il fallait des recherches ardues dont on imagine difficilement de l'extérieur l'étendue et le caractère fastidieux. Pour une publication papier (et pas seulement d'ailleurs), seule une édition rigoureusement annotée présente un réel intérêt à l'heure où tant de documents sont disponibles à l'état brut sur la Toile, et donnent lieu à des 
interprétations souvent tendancieuses ou tout simplement sans rapport avec le contexte dans lequel ils ont été rédigés.

3 Comme chez nombre de ses contemporains, les formules infantilisantes, les jugements à l'emporte-pièce émis sur un ton de supériorité à propos des défauts et qualités supposés des races dans les Balkans ne manquent pas dans les écrits de Lejean. À propos des Valaques, par exemple : ceux du nord du Danube, les Roumains, et non ceux de Macédoine, d'Epire et de Thessalie, les Aroumains (qu'il appelle, p. 372, Valaques du Sud ou encore Zinzares). Dans une lettre écrite en juin 1857, il en dresse un tableau haut en couleur assez déconcertant de prime abord :

Quelle singulière nation! Des enfants qui se croient des hommes, mais du moins des enfants sympathiques. Les Serbes, que j'admire bien davantage, sont dignes mais un peu roides. Les Roumains sont les Français du Danube, avec une certaine ingénuité en plus ; seulement ils tiennent à ne pas être ingénus. Leur cordialité est charmante et leur dévouement sincère. Le sentiment ne leur manque pas ; c'est la solidité, le sérieux, la moralité qui leur manquent. Je leur dis quelquefois: Vous êtes des enfants, et déjà vous êtes vieux et gangrenés jusqu'à la moelle. Ils me répondent: C'est le fait des phanariotes. Eh! Morbleu, laissez-là les phanariotes, et corrigezvous! J'aime quelques individus mais pas la masse. Il m'a paru que les paysans valent mieux, si affaissés qu'ils soient; quant à l'aristocratie c'est une étable d'Augias. Entrez dans un salon, et vous pouvez dire tout d'abord: neuf sur dix de ces hommes sont des voleurs; neuf sur dix de ces femmes sont des... ou le seront. Une frivolité, une légèreté, une paresse, un luxe inouïs, et, pour suffire à des dépenses folles, des ressources honteuses ou l'exploitation impitoyable du klakasch (paysan corvéable). Il est vrai que la boyarie confiant aux Tziganes bohémiens l'éducation de ses enfants et la conduite de ses voitures, on voit d'où sort chaque génération. (p. 268)

Les Valaques ne sont évidemment pas les seuls à avoir droit à de tels raccourcis désobligeants, et les généralisations sont d'un goût douteux, même quand elles se veulent bienveillantes comme dans le cas des pacifiques laboureurs bulgares et des vaillants guerriers serbes.

Pour être gênante rétrospectivement, la rhétorique centrée sur la psychologie des peuples qui abonde dans les écrits des voyageurs, géographes et diplomates occidentaux parcourant le Sud-Est européen ne devrait pas nous faire perdre de vue le fait que c'est à ces écrits que nous devons pour l'essentiel les maigres connaissances que nous détenons sur la région et ses habitants à l'époque. En critiquant le «balkanisme» forgé de l'extérieur, on devrait rappeler le silence qui a longtemps prévalu dans ces pays à propos de leur histoire, de la vie quotidienne, des coutumes... Les raisons d'un tel mutisme sont complexes, et le poids de l'administration ottomane est sans doute pour beaucoup, mais ce n'est pas une raison pour en faire abstraction. C'est le reproche que je ferais à Maria Todorova, auteure d'un ouvrage par ailleurs incontournable dont on ne peut que saluer la traduction en français ${ }^{2}$. L'arrivée des États-nations allait changer la donne, en favorisant l'émergence sur place d'intellectuels et d'experts souvent de valeur et d'un public désireux de connaître l'histoire et les réalités de son pays. Dans un sens, on ne peut que s'en féliciter, tout en déplorant l'usage nationaliste de l'histoire forgée dans ces conditions. Le peu d'attention accordé à l'histoire et aux réalités communes de la région constitue une autre conséquence de cette évolution, plus rarement prise en compte. Aussi, dès lors qu'il s'agit de l'histoire de l'ensemble de la région, surtout pendant la période qui précède l'entrée en lice des États nationaux, à moins de se contenter des versions proposées par chacune des histoires nationales, on est conduit à recourir aux données, 
descriptions et analyses fournies par des auteurs extérieurs. Cela vaut jusqu'à une date récente, les choses étant justement en train de changer, y compris grâce à un livre comme L'imaginaire des Balkans de M. Todorova. Née en Bulgarie et diplômée de l'Université de Sofia, cette auteure est bulgare, certes, mais son livre a été écrit en anglais aux États-Unis et procède d'une problématique occidentale, tout au moins au départ, initiée par les travaux sur l'orientalisme du Palestinien Edward Said qui enseignait également aux États-Unis et s'adressait à un public anglophone ${ }^{3}$.

On ne saurait donc en vouloir à Lejean pour ses écarts stylistiques et, pour revenir au passage que nous venons de citer in extenso, on peut faire remarquer que, pour être déconcertant, il n'est pas moins saisissant pour ce qui est de la critique de l'« aristocratie » roumaine. S'il épouse certains préjugés de son temps, Lejean, qui était issu de la paysannerie bretonne, est aussi un observateur attentif et fait preuve d'une extrême honnêteté intellectuelle dans ses descriptions, précieuses, notamment lorsqu'elles portent sur le monde bulgare.

7 Pour compléter le tableau des Valaques, rappelons, avec Marie-Thérèse Lorain (p. 11), que Lejean a dû côtoyer à Paris les «nobles proscrits" roumains de la révolution de 1848 dans les cours de Jules Michelet au Collège de France (les frères Bratianu, Constantin Rosetti, Ion Ghica) et qu'il s'est appuyé parfois sur les travaux des « savants indigènes » de Iasi et de Bucarest (Mihail Kogalniceanu, August Troboianu Laurian, Cezar Boliac) mais aussi de Belgrade (p. 48), ce qui fait de lui un «passeur» entre «la jeune recherche scientifique balkanique et les cercles savants parisiens", pour reprendre les mots de Bernard Lory (p. 61 et 437).

8 Sur les routes des Balkans, d'une découverte à l'autre ${ }^{4}$, Lejean poursuivait un but assez précis: établir une carte ethnographique de la Turquie européenne, c'est-à-dire combler les taches blanches des cartes établies par ses prédécesseurs. Élysée Reclus l'a reprise dans sa Géographie universelle en sorte que cette carte en couleur a marqué durablement la perception de la répartition des populations dans les Balkans et joué un rôle considérable lors des négociations portant sur le partage des anciennes possessions de l'Empire ottoman. Dans sa postface, Bernard Lory écrit :

Sans le savoir, avec le désir naïf de classification de l'érudit de cabinet, Lejean contribue au processus de discrimination nationale qui va déchirer les Balkans jusqu'à nos jours. Il fournit des arguments aux tenants de l'État-nation qui militent pour des territoires ethniquement homogènes et qui n'hésiteront pas à convertir, dénationaliser, déporter ou massacrer ceux qui ne rentrent pas dans le grand dessin unificateur. Par ignorance, Lejean a fait figurer de grandes zones monochromes sur sa carte, qui laissent entendre que des États homogènes sont facilement réalisables dans les Balkans. (p. 440-441)

9 Cette conclusion est peut-être en peu sévère pour le Lejean. En effet, en dessous de la carte publiée par Elysée Reclus figure la légende suivante : «Cette carte ne peut avoir qu'une valeur approximative. La plupart des populations de races et de langues diverses sont entremêlées et non juxtaposées. $»^{5}$ 


\section{NOTES}

1. Lejean (Guillaume), Voyages dans les Balkans, 1857-1870, textes édités et présentés par MarieThérèse Lorain et Bernard Lory. - Paris : Non lieu, 2011. - (493 p.) : ill. - ; 24 x 16 cm. - (Via Balkanica)

2. Todorova (Maria), L'imaginaire des Balkans, trad. Rachel Bouyssou, Paris : Editions EHESS, 2011. 3. Paru en 1997, Imagining the Balkans été traduit en 1999 en bulgare et en serbe, en 2000 en roumain et en grec, en 2001 en slovène et en macédonien, en 2003 en turc et en 2006 en albanais.

4. Il est, par exemple, le premier à faire état de l'existence des Gorani, musulmans slavophones du Kosovo, qu'il a rencontrés lors de la traversée du Šar en 1869 (pp. 373-374).

5. Nouvelle Géographie universelle. 1, L'Europe méridionale, Paris : Librairie Hachette, 1876. 